\section{References}

Bhui, K. (2001) Epidemiology and social issues. In Psychiatry in Multicultural Britain (eds D. Bhugra \& R. Cochrane), pp. 49-74. London: Gaskell.

Chatterjee, S., Patel, V., Chatterjee, A., et al (2003) Evaluation of a community-based rehabilitation model for chronic schizophrenia in rural India. British Journal of Psychiatry, 182, 57-62.

Edgerton, R. B. \& Cohen, A. (1994) Culture and schizophrenia: the DOSMD challenge. British Journal of Psychiatry, $164,222-231$.

Halliburton, M. (2004) Finding a fit: psychiatric pluralism in South India and its implications for WHO studies of mental disorder. Transcultural Psychiatry, 41, 80-98.

H igginbotham, N . \& Marsella, A. J. (1988) International consultation and the homogenization of psychiatry in Southeast Asia. Social Science and Medicine, 27, 553-561.

Jablensky, A., Sartorius, N., Ernberg, G., et al (1992) Schizophrenia: manifestations, incidence and course in different cultures. Psychological Medicine (monograph supplement 20).

Kulhara, P. (1994) Outcome of schizophrenia: some transcultural observations with special reference to developing countries. European Archives of Psychiatry and Clinical Neurosciences, 244, 227-253.

Leff, J., Sartorius, N., Jablensky, A., et al (1990) The International Pilot Study of Schizophrenia: five year follow up findings. In Search for Causes of Schizophrenia, Vol. II (eds H. Haffner \& W. F. Guttaz). Berlin: Springer.

Lewis, A. (1953) Health as a social concept. British Journal of Sociology, 4, 109-124.

Peet, M. (2004) International variations in the outcome of schizophrenia and the prevalence of depression in relation to national dietary practices: an ecological analysis. British Journal of Psychiatry, 184, 404-408.

Radford, M. D. (2000) Getting the 'nuts' and the 'bolts' together. In At the Core of Mental Health Practice: Key Issues for Practitioners, Managers and Trainers (ed. D. Bailey). London: Pavilion.

Thara, R., Padmavati, R. \& Srinivasan, T. N. (2004) Focus on psychiatry in India. British Journal of Psychiatry, 184, 366-373.

Tooth, B., Kalyansunderaram, V. \& Glover, H. (1997) Recovery from Schizophrenia: A Consumer Perspective. Centre for Mental Health N ursing Research, Q ueensland University of Technology.

World Health Organization (1979) Schizophrenia: An International Follow-Up Study. Chichester: Wiley.

\title{
Traditional Chinese medicine in psychiatric practice in Singapore
}

\author{
Ee Heok Kua MBBS MD FRCPsych ${ }^{1}$ and Chay Hoon Tan MBBS PhD MMed (Psych) ${ }^{2}$
}

${ }^{1}$ Professor and Senior Consultant Psychiatrist, Department of Psychological Medicine, National University of Singapore, National University Hospital, 5 Lower Kent Ridge Road, Singapore 119074,

email pcmkeh@nus.edu.sg

${ }^{2}$ Associate Professor and Consultant Psychiatrist, Department of Pharmacology, National University of Singapore

round three-quarters of the Singaporean population are of $C$ hinese ethnic origin, and so traditional Chinese medicine is the most widely practised non-Western system of healthcare on the island.

Traditional Chinese medicine was first recorded some 2500 years ago in the ancient textbook of medicine Classics of Internal M edicine (H uang-Di N eijing), a collection of dialogues between the Yellow Emperor and his court physician, Ch'i Po. Written during the era of the Warring States (476-221 BCE), it showed an awareness of emotional and psychological factors in the causality of physical illness. A mind-body nexus rather than dichotomy was emphasised.

In Chinese culture, theories of health and illness are embedded in customs and religious philosophies. Religious beliefs and spirituality are therefore important issues in psychiatric practice. Since ancient times, religious practice and healing have been closely associated, and healing has often been performed by priests. $W$ ith advances in scientific knowledge, the healing role has now largely been taken over by doctors, but priests or traditional healers are still consulted first in many Chinese communities.

The basis of traditional Chinese medicine is the belief that there is a finely balanced relationship between bodily functions and emotions. This belief is built on the concept of 'yin-yang', a bipolarity that is opposite and complementary. The yin represents coldness and yang warmth. When this homoeostasis is disrupted, physical or mental illness can result. The prescription of herbal medicine or the administration of acupuncture attempts to restore the imbalance of the yin and yang. Besides physical treatment, it is the shared cultural beliefs of the healer and the patient and the relationship between these two that are pivotal in recovery.

\section{Health-seeking behaviour}

Family and cultural beliefs often determine illness behaviour and the health-seeking tendency of the patient. In a study of the illness behaviour of 100 Chinese patients referred consecutively to the psychiatric clinic of the $\mathrm{N}$ ational University Hospital in Singapore, it was found that 36 had also consulted traditional healers (Kua et al, 1993). Most of these patients were suffering from depression, anxiety or schizophrenia. It is common in the Chinese community to consult both the traditional healer and the psychiatrist.

The possession trance is a common culture-related phenomenon in Singapore and many other countries in Asia. The characteristic features have been reported by Kua (1986). Because possession trance is not deemed an illness in the community, a traditional healer is often
Family and cultural beliefs often determine illness behaviour and the healthseeking tendency of the patient. In a study of the illness behaviour of 100 Chinese patients referred consecutively to the psychiatric clinic of the National University Hospital in Singapore, it was found that 36 had also consulted traditional healers. 
consulted. This socially sanctioned behaviour is recognised as a sign of distress and evokes the appropriate family response - support and sympathy. Those with the condition are treated with respect because they are perceived to be favoured by a deity. Treatment by the traditional healer lacks the stigma associated with referral to a mental hospital. In a follow-up study of depression among elderly Chinese people, it was noticed that many did not seek treatment in a hospital or out-patient clinic but preferred traditional medicine (Kua, 1993).

\section{Physical treatment}

In traditional $\mathrm{C}$ hinese medicine, herbs and acupuncture are the main methods of treatment for psychiatric conditions. Acupuncture has been used to relieve headache, insomnia, depression and chronic pain. A study by Luo et al (1997), from the Beijing Institute of Mental Health, showed that acupuncture was as effective as amitriptyline in the treatment of depression. Extracts of herbs like Astragalus membranaceus, Angelica sinensis and W ikostroemia chamedaphre have been claimed to be useful in the treatment of both depression and insomnia (Liu, 1981; Tien, 1985).

For many centuries, Chinese people have known about the effects on longevity of the consumption of Ginkgo biloba extract (from the leaves of the Ginkgo tree). However, there is a paucity of scientific data to support this assertion, although all round the world Ginkgo biloba extract is sold over the counter in pharmacies for the prevention of memory impairment in late life. De Smet (2002) reviewed the scientific evidence on the Ginkgo biloba extract as an antioxidant.

In traditional Chinese medicine there is also an emphasis on exercise as part of a healthy lifestyle to restore the yin-yang equilibrium. Tai-chi or kung-fu is encouraged as a form of exercise during the recuperation phase of physical or mental illness. It is interesting to note that many traditional healers and priests (e.g. the Shaolin monks) are themselves experts in kung-fu, which is taught in temples as an art of self-defence.

\section{In Western \\ psychotherapy, \\ the focus is on the individual}

struggling with

biological urges

and social

constraints. In

Chinese culture,

the emphasis is on

the individual as a member of a

family. heightened if it is also known that he or she is an expert in tai-chi or kung-fu.

In the clinic, healers examine the patient largely through observation and feeling the pulse. There is minimal verbal communication and they explain to patients the state of their yin and yang. The healer explains the symptoms using the belief systems the patient is familiar with. To the depressed patient, symptoms of poor sleep, loss of concentration and listlessness are interpreted as due to a 'weakness of mental energy'. It is difficult to explain or understand the patient's neurotic problems in Western psychoanalytic terms like the ' $O$ edipus complex' or 'ego strength'.

The Japanese Morita model of psychotherapy (named after Shoma Morita, 1874-1938) is influenced by Buddhism. During treatment, there is a phase of disengagement from the precipitating factor (e.g. leaving an intolerable relationship or situation), introspection (rethinking the issues and planning different strategies in problem solving), conflict resolution (with suggestions by the healer to overcome the impasse) and finally the phase of acceptance of reality and solutions. The paternal transference on the healer is likened to the master-student relationship, as in martial arts training. The healer's suggestions help patients to accept themselves and to internalise the healer's wisdom. A healthy diet with herbal supplements and regular exercise or martial arts training are integral to the recovery process.

\section{Conclusion}

In the provision of psychiatric care in the East, the role of the traditional healer is gradually being acknowledged. In Indonesia, psychiatrists work closely with traditional healers and organise training programmes to help them identify patients with psychosis, to ensure their early referral to the hospital for treatment with antipsychotic medications. This collaborative effort is especially vital in countries where there is a perennial shortage of trained psychiatrists and other mental health professionals.

In a study of depression in elderly Chinese people (Kua, 1993), many patients preferred traditional healers partly because of their accessibility (the clinics were usually near their homes) and because the fees were affordable. Consulting a healer also avoided the stigma of being labelled a 'mental patient'.

W ith globalisation, the issue of 'cultural intelligence', as described by Earley and Mosakowski (2004) from the London Business School, is crucial as economic expansion crosses national boundaries and continents and so also very different cultures and customs. Modern psychiatry grew out of Western Europe and the U nited States. Most psychiatrists in the East are schooled in Western ideas of psychiatric practice. There is much to learn from the psychotherapeutic techniques of traditional healers in the management of psychiatric disorders. Fundamental in the therapeutic relationship is trust, and an understanding of the cultural belief system is a sine qua non. 
Kua, E. H., Chew, P. H. \& Ko, S. M. (1993) Spirit possession and healing among Chinese psychiatric patients. Acta Psychiatrica Scandinavica, 88, 447-450.

De Smet, P. A. (2002) Herbal remedies. New England Journal of Medicine, 347, 2046-2056.

Earley, P. C. \& Mosakowski, E. (2004) Cultural intelligence. $H$ arvard Business Review, $O$ ctober, 139-146.

Kua, E. H. (1986) A cross-cultural study of the possessiontrance in Singapore. Australian and $\mathrm{New} Z$ Zealand Journal of Psychiatry, 20, 361-364.

Kua, E. H. (1993) The depressed elderly Chinese living in the community: a five-year follow-up study. International Journal of Geriatric Psychiatry, 8, 427-430.

Liu, X. H. (1981) Psychiatry in traditional Chinese medicine. British Journal of Psychiatry, 138, 429-433.

Luo, H. C., Zhao, X. Y. \& Meng, F. Q. (1997) Clinical research progress of electro-acupuncture treatment on common mental disorders. Abstracts of World Psychiatric Association Regional Meeting, Beijing, China, 7-10 O ctober. Beijing: Institute of Mental Health.

Tien, J. K. (1985) Traditional Chinese beliefs and attitudes toward mental illness. In Chinese Culture and M ental Health (eds W. S. Tseng \& D. Y. H. Wu). O rlando, FL: Academic Press.

\section{Mental health in Mongolia}

\section{S. Byambasuren MD PhD and G. Tsetsegdary MD PhD}

Policy and Coordination Department, Ministry of Health, Government Building 8, Olympic Street 2, Ulaanbaatar, email tsetsegdary@yahoo.co.uk

ongolia is a country with an approximate area of 1.5 million $\mathrm{km}^{2}$. Its population is 2.5 million, nearly $90 \%$ of whom are ethnically Mongolian. Khalkh Mongols form the largest subgroup (approximately $79 \%$ of the population); the next largest subgroup is the Kazakhs (5.3\%), followed by smaller groups such as Tuvins, U zbeks, U ighurs, Russian and Chinese. The population is young, with $35.9 \%$ under the age of 15 years. The official language is Mongolian. Just under half the population live in rural areas and around a fifth live a nomadic life. About $80 \%$ of the land area is suitable for agriculture, mostly for animal husbandry.

According to the statistical data, gross domestic product (GDP) per capita was 500744 tugriks (approximately U S\$420) in 2002. In 2000 some $36 \%$ of the population were living below the poverty line, and in 2002 the unemployment rate was 3.4\%. Education is obligatory for all children aged between 8 and 15 years and the literacy rate is $98 \%$ for men and $95 \%$ for women.

Life expectancy at birth is 63.5 years (2002). The infant mortality rate is 23.5 per 1000 live births (2003), and the maternal mortality rate is 110 per 100000 live births (2003). Socio-economic changes such as poverty, unemployment, the destabilisation of family structure, natural and man-made disasters, changes to traditional culture and lifestyle, and urbanisation are major factors affecting mental health. These current social changes result in suicide, street children, acts of violence and substance misuse, especially alcoholrelated problems.

\section{Epidemiological research}

According to the results of an epidemiological survey conducted between 1976 and 1984, the prevalence of mental disorders per 1000 population varied widely across the country, from 9.8 in Altai (a mountainous region), to 13.1 in Khangai and Khentii (both also mountainous regions), 18.3 in Dornod (a steppe region), 23.5 in the Gobi (a desert region) and 24.0 in the capital, U laanbaatar (Byambasuren, 2000). These figures do not include those people with less severe psychological or psychosocial problems. Epidemiological studies on the prevalence of suicide (Byambasuren et al, 2003) and schizophrenia (Khishigsuren et al, 2004) have been conducted. According to this research, the number of suicides in U laanbaatar increased nearly threefold between 1992 and 2002, to reach 3.0 per 10000 population. The prevalence of schizophrenia in U laanbaatar is 0.97 cases per 1000 .

\section{Mental health legislation and the National Mental Health Programme}

Mental health legislation passed in 2000 and the $\mathrm{N}$ ational Mental Health Programme of 2002 have been the key elements of a reform of mental health care in Mongolia.

The legislation covers all aspects of mental health, including:

O policy and principles

o the duties of state organisations, business entities and individuals

o mental health promotion

o the structure, management and financing of mental healthcare services

O the rights of people with mental illness

o involuntary admission

0 the provision of security and social welfare assistance to people with mental illness.

The aim of the $\mathrm{N}$ ational Mental $\mathrm{H}$ ealth Programme is to reduce the prevalence of mental and behavioural
Contributions to the country profile section are welcome: please contact Shekhar

Saxena (email saxenas@who.int).

The number of suicides in

Ulaanbaatar increased nearly threefold between 1992 and 2002, to reach 3.0 per 10000 population. The prevalence of schizophrenia in Ulaanbaatar is 0.97 cases per 1000. 